\title{
New Candidate Delivery System for Alzheimer's Disease: Deferoxamine Nanogels
}

\author{
Hajar Ashrafi 1, *(D) , Amir Azadi 1 ${ }^{(D)}$, Soliman Mohammadi-Samani 1 ${ }^{(D)}$, Mehrdad Hamidi 2,*(D) \\ 1 Department of Pharmaceutics, School of Pharmacy, Shiraz University of Medical Sciences; hashrafi@ sums.ac.ir; \\ aazadi@sums.ac.ir; smsamani@sums.ac.ir; \\ 2 Department of Pharmaceutics, Faculty of Pharmacy, Zanjan University of Medical Sciences; hamidim@ zums.ac.ir; \\ * Correspondence: hashrafi@sums.ac.ir (H.A.); hamidim@ @ums.ac.ir (M.H.);
}

Scopus Author ID 54415320600, 12793079400

Received: 12.05.2020; Revised: 1.06.2020; Accepted: 2.06.2020; Published: 6.06.2020

\begin{abstract}
This study presents hydrogel nanoparticles made of chitosan and tripolyphosphate via ionotropic gelation technique to evaluate their potential for the association of deferoxamine. Since it has been shown that iron chelation therapy can be useful in the treatment of some neurodegenerative diseases such as Alzheimer's, in this study, we attempted to evaluate the in vitro characteristics of deferoxamine-loaded nanogels for this purpose. Chitosan-based nanogels were prepared and optimized in terms of size by a Taguchi Orthogonal Array design. The spherical structure nanoparticles showed a uniform particle size of $63 \pm 2 \mathrm{~nm}$ with loading amount, Loading efficiency, and loading ratio of $8.48 \% \pm 0.021,26.1 \% \pm 0.63$, and $2.66 \% \pm 1.88$ respectively in optimum conditions. Further study revealed that this nanoparticulate system can be a suitable carrier for the entrapment and control release of small molecules such as deferoxamine.
\end{abstract}

Keywords: Hydrogel nanoparticles; Chitosan; deferoxamine; Ionotropic gelation method; Iron binding capacity.

(C) 2020 by the authors. This article is an open-access article distributed under the terms and conditions of the Creative Commons Attribution (CC BY) license (https://creativecommons.org/licenses/by/4.0/).

\section{Introduction}

Nanoparticles as a new attempt in the drug delivery system with their capability in small and controllable sizes have the extended multiplicity of feasibility for controlling their structures and functions for the goal of targeting and also for long-circulating delivery $[1,2]$. Among this wide variety of nanoparticles, hydrogel nanoparticles due to their exceptional characteristics, like wonderful hydrophilicity, biocompatibility, biodegradability, and the relative ease of manner and handling, have gained new attention in recent years for drug delivery $[3,4]$. These hydrophilic nanoparticles can evade the reticuloendothelial system (RES) and remain in circulation for a long time [5]. Various types of hydrogel nanoparticles are prepared and characterized for drug delivery, which has been mostly classified based on the type of polymeric materials used in the preparation of the nanoparticles. Among polymers, chitosan is one of the plentiful, renewable, non-toxic, and biodegradable carbohydrate polymers, which is one of the most polymers used in the preparation of hydrogel nanoparticles $[6,7]$. Different methods have used to prepare chitosan particulate systems. However, the selection of any of the methods depends upon the nature of the active molecule as well as the type of delivery device [8]. Ionotropic gelation method is an available, straightforward, and mild method that is based on the making cross-linkage between oppositely charged polymers by the polyanions in the presence of the drug to be loaded [9]. Tripolyphosphate (TPP) is a 
polyanion, which can interact with the cationic chitosan by electrostatic forces $[10,11]$. In this study, we used chitosan polymer and tripolyphosphate to prepare nanogels containing deferoxamine (DFO).

Iron chelation therapy with DFO is one of the most ways to manage iron over storage and improve the well-being and life prospect of Alzheimer's (AD) [12] and Parkinson's disease $[13,14]$, thalassemia [15, 16], wound healing [17], tissue regeneration [18] and so on. It has suggested that DFO, an FDA approved iron chelator, could be a potential treatment for various neurodegenerative disorders such as $\mathrm{AD}$ [12]. $\mathrm{AD}$ is one of the common chronic neurodegenerative diseases, which affects nearly 50 million people in the world. Currently, only the two types of drugs used to relieve $\mathrm{AD}$ symptoms only are Acetyl Cholinesterase Inhibitors (AChEI), and N-methyl-D-aspartic acid (NMDA) are drugs that do not stop or slow the progression of the disease [19-21]. There are several small molecules among the experimental drug classes. Their effects have studied for molecular responses associated with the progression of $\mathrm{AD}$, which includes Paroxetine [22], Indomethacin [9, 23], Lithium Carbonate [24], N-acetylcysteine [25], intravenous immunoglobulin [26, 27], Carvedilol [28], and DFO [29].

It has shown that the neurotoxicity of amyloid $\beta$ peptide in $\mathrm{AD}$ is enhanced by iron, which is present in amyloid plaques in $\mathrm{AD}[30,31]$. Amyloid $\beta$ peptide is a predominant histopathological lesion in AD [32,33]. It has reported that the effects of DFO on neuronal activity, synaptic plasticity, and BDNF may be significant in reducing memory impairment in AD models [34].

DFO is a potent iron and aluminum chelating agent approved by the FDA and used the treatment of iron overload caused by blood transfusions. DFO has had many benefits in treating $\mathrm{AD}$ disease for patients. Daily doses of intramuscular injection of this drug can reduce inflammation as well as slowing the progression of AD by reducing tau and also reducing free radical production [32]. Also, DFO inhibits tau protein through GSK-3 $\beta$ hyperphosphorylation. Besides, the DFO has reduced cognitive decline problems in APP / PS1 transgenic mice and non-transgenic mice and has increased memory in Alzheimer's mice [35].

Finally, DFO is effective in improving AD by inhibiting $\mathrm{A} \beta$ deposits and creating apoptosis in the brains of APP / PS1 transgenic mice [36]. Since DFO has a very short half-life and not orally bioavailable, it is administered by either SC or IV infusion for 8-12 hours per day up to six times per week [37]. However, poor patient compliance with this strict parenteral DFO regimen may result in negative iron balance, which underlines the need for alternative drugs and novel methods of drug administration [37, 38].

So, as mentioned, iron chelators like DFO can be good options for further research on improving AD. In a recent study, a sample of DFO-containing hydrogels used for this purpose, which was able to have effective results. Nevertheless, more research is needed.

Therefore, in this study, we tried to be able to design a suitable drug delivery system based on nanogels that contains DFO and to deliver the drug to the brain using an intravenous injection or intranasal method. As will be shown in the following results, this method has been able to increase the drug's ability to bind iron, which is more promising in improving AD.

Since the polymeric system use in this study is a natural one with well-documented biocompatibility and hydrophilicity, we expect the resulting nanogels to be well-tolerated that the size and surface potential range achieved allow it to do so. 


\section{Materials and Methods}

\subsection{Materials.}

Chitosan (minimum deacetylation degree of $85 \%$, Sigma-Aldrich, USA, Lot. no. 212F498-89), Penta-Sodium tripolyphosphate (Merck, Germany, Lot. No. K36643499-742) and Deferoxamine mesylate (Desferal ${ }^{\circledR} 500 \mathrm{mg}$ vials, Novartis Pharma AG, Basle, Switzerland) were purchased locally. Other chemicals and reagents were from decent purity grades and all purchased locally.

\subsection{Preparation of hydrogel nanoparticles.}

In this study, the ionotropic gelation method involving the polycation polymer, chitosan, and a polyanionic counter ion, tripolyphosphate, was adopted and used for the preparation of DFO loaded hydrogel nanoparticles intended for drug delivery [1]. The method has already been optimized concerning all parameters in terms of their single as well as combination effects on the finally resulted in particle sizes using a Taguchi orthogonal array design $[39,40]$. Briefly, in the optimum condition, the polyanion TPP solution (5\% (w/v)) was added to polysaccharide chitosan solution $(0.3 \%(\mathrm{w} / \mathrm{v})$ in acetate buffer of $0.67 \mathrm{M}, \mathrm{pH}=4)$ in a dropwise manner, with the volume ratio of 1:8 (TPP:CS) over 2 minutes' time period with constant magnetic stirring $(1500 \mathrm{rpm})$ at $25^{\circ} \mathrm{C}$. The stirring continued for $20 \mathrm{~min}$. The most effective factors on the size of nanogels were, by rank, TPP/chitosan volume ratio, chitosan concentration, temperature, addition time of TPP solution to chitosan solution, and TPP concentration.

\subsection{Preparation of DFO loaded hydrogel nanoparticle.}

For the association of DFO with chitosan nanoparticles, DFO in the concentration of $3.125,6.25,12.5$, and $15 \mathrm{mg} / \mathrm{ml}$ dissolved in chitosan solution used for nanoparticle preparation. After mixing with the TPP solution during the preparation procedure, the optimum drug concentration in terms of the loading parameters will be found. The reminding part of the preparation method carried out proportional to the previous section. Triplicate samples analyzed in each case.

\subsection{In vitro characterizations of DFO-loaded nanoparticles.}

For the measurement of particle size and poly-dispersity (size distribution), Particle Size Analyzer (Shimadzu, model SALD-2101, Japan) used, which measured the sizes based on the Laser Diffraction techniques. Particle size measurement was performed manually using a quartz cell. Samples diluted to appropriate concentrations with distilled/filtered water. Each value averaged from three parallel measurements. Since the surface zeta potential of the drugloaded nanoparticles is one of the other significant determinants of the biofate of the carriers, the zeta potential of the optimized DFO-loaded nanoparticles was recorded on a Zetasizer ${ }^{\circledR}$ 3000-HS, (Malvern Instruments, UK), working based on Photon Correlation Spectroscopy (PCS) technique. Besides, morphological examination of the hydrogel nanoparticles performed by transmission electron microscopy (TEM) (Philips, model CM10). Samples were immobilized on copper grids, dried at room temperature and then, examined using a TEM without being stained. Then for chemical characterization, Fourier transforms infrared spectroscopy (FT-IR) used. The IR spectra of lyophilized chitosan nanoparticles, both DFO- 
loaded and unloaded nanoparticles, were taken in $\mathrm{KBr}$ pellet using Shimadzu FT-IR spectrophotometer (model 8000 series, Japan) in comparison to free DFO samples.

\subsection{Drug assay.}

The ferrioxamine complex between DFO and ferric iron was used for the detection of DFO. DFO Solutions are colorless, but ferrioxamine has an orange-red color, which, depending on its concentration, may be apparent to the naked eye. In this method, a stock solution of 150 $\mathrm{mg} / \mathrm{mL}$ of DFO prepared in distilled water, and the concentrations of $2.5,5,10,15 \mathrm{mg} / \mathrm{mL}$ prepared with drug-free nanoparticles suspensions with the same concentration prepared routinely during the studies. Then, the spiked samples diluted to 1:10, and the particles then destroyed by adding 1:1 acetonitrile. To $2 \mathrm{ml}$ of the samples prepared as described, $3 \mathrm{~mL}$ of Ferric chloride solution, prepared by dissolving $3.35 \mathrm{~g}$ of ferric chloride in dilute hydrochloric acid ( 1 in 100), was added and the resulting solution is diluted to $25 \mathrm{ml}$ with distilled water and after, vortex-mixing for 1 minute, the absorbance of the samples determined at $485 \mathrm{~nm}$ against the corresponding drug-free bland undertaking the same procedure on UV spectrophotometry.

\subsection{DFO loading parameter.}

The total concentration of the drug in the nanoparticles determined by destroying the nanoparticles by adding a 1: 1 ratio of acetonitrile to the dispersion and then diluting the nanoparticles in distilled water in a ratio of 1 to 10 . Finally, the drug concentration was determined using a pre-developed UV spectrophotometric method. The unloaded drug concentration, on the other hand, was determined by the separation of nanoparticles from the aqueous medium containing unloaded drug via filtration through a $50 \mathrm{~nm}$ membrane filter (Millipore ${ }^{\circledR}$, Bedford, USA) and, then, measurement of free drug amount in the supernatant using the developed UV method.

The loaded amount (LA) of DFO in nanoparticles determined as:

$\mathrm{LA}=($ Total DFO concentration - Free DFO concentration $) \times$ Sample volume

The loading ratio (LR) and loading efficiency (LE) determined as:

$\mathrm{LR}=[($ Total DFO concentration-unloaded DFO concentration $) /$ Total DFO concentration $]$ $\times 100$

$\mathrm{LE}=(\mathrm{LA} /$ Total $\mathrm{DFO}$ added during the loading procedure $) \times 100$

\subsection{In-vitro drug release.}

To exploit the release kinetics of DFO from nanoparticles, $10 \mathrm{ml}$ of DFO-loaded nanoparticles suspensions, as obtained, was diluted to $100 \mathrm{ml}$ of PBS (Phosphate Buffer Saline; $\mathrm{pH}=7.4$ ), and the suspension was mixed thoroughly by several gentle inversions. The mixture then divided into fifteen $5-\mathrm{ml}$ portions in $15 \mathrm{ml}$ polypropylene tubes. The samples shake vertically $(15 \mathrm{rpm})$ while incubated in $37^{\circ} \mathrm{C}$ using a vertically shaking incubator designed and assembled in-house. At the beginning of the test and 2, 4, 8, 12, 24, 48, $72 \mathrm{hrs,} \mathrm{also} \mathrm{at} \mathrm{1,} \mathrm{2,} \mathrm{and}$ 3 weeks and 1, 2, and 3 months intervals, one of the aliquots harvested from which $0.5 \mathrm{ml}$ was used for particle destruction by the addition of $0.5 \mathrm{ml}$ of acetonitrile. Also, $4 \mathrm{ml}$ of the remaining samples filtered with a $50 \mathrm{~nm}$ membrane filter. The final $0.5 \mathrm{ml}$ portion of the filtrate was mix with $0.5 \mathrm{ml}$ of acetonitrile. Then, the free and total drug concentrations in samples were determined spectrophotometrically at $485 \mathrm{~nm}$. These experiments carried out in triplicate, and the percent of drug release in each case was determined. 


\subsection{Stability of DFO loaded nanoparticles.}

This test carried out to evaluate the stability of the sizes of the DFO-loaded nanoparticles with time in two different conditions: ambient and refrigerated temperatures. In brief, $20 \mathrm{~mL}$ of freshly prepared DFO-loaded nanoparticle suspensions, as obtained, was divided into two $10-\mathrm{ml}$ portions in $15 \mathrm{ml}$ polypropylene tubes: one tube placed in a lab refrigerator $\left(2-8^{\circ} \mathrm{C}\right)$ and the other one kept at room temperature $\left(22-28^{\circ} \mathrm{C}\right)$. At the start time of the test and 1, 2, 4, 8, 12, 24, 48, $72 \mathrm{hrs}, 1,2$, and 3 weeks and also at 1, 2, 3 months intervals, the particle size and particle size distribution of both samples monitored. Each value averaged from three parallel measurements.

\subsection{Preparation of nan powder.}

For the preparation of a suitable nanopowder form of nanogels, cryoprotectant excipients such as glucose, mannitol, sucrose, glycerin and tween 80 which are a necessary part of the freeze-drying procedure, were added to the nanoparticle dispersions, each at concentrations of $1 \%, 3 \%$, and $5 \%$. The nanoparticle dispersions were frozen for a minimum of 12 hours at $-32{ }^{\circ} \mathrm{C}$ and, then, dried at $-55{ }^{\circ} \mathrm{C}$ and $0.5 \mathrm{kPa}$ for 24 hours (Christ, $\alpha 1-4 \mathrm{LD}$ plus, Germany). Finally, all dried samples were reconstituted in distilled/filtered water to appropriate concentrations, and their sizes were evaluated by particle size analyzer, as mentioned.

\subsection{Yield of nanoparticles.}

The process yield, as well as the overall recovery of the DFO-loaded nanoparticle preparation process, was determined after the separation of the nanoparticles from out-of-range waste materials. Aqueous suspensions resulting from the nano-gelation procedure, as described earlier, were centrifuged at $3000 \mathrm{~g}$ for $5 \mathrm{~min}$ and, then, nanodispersion (i.e., supernatant), as well as the waste material (i.e., centrifuge pellet), were freeze-dried separately until reaching a constant weight of each portion, while using glucose as cryo-protectant in the concentration of $5 \%$ w/v. Nanoparticles yield (NPY), by convention, was defined as:

NPY $=[($ Dried weight of nanodispersion - the nominal weight of cryoprotectant added $) /$ Total nominal masses added during the procedure $] \times 100$

Also, the overall recovery (accuracy of the processed determined as:

Recovery of the process $=($ Total recovered mass $/$ Total incoming mass $) \times 100$

\subsection{Iron Binding Capacity.}

To determine the iron-binding capacity (IBC) of DFO while loaded in nanoparticles was measured and compare it with the IBC of the free drug as the functional effect of drug binding in nanoparticles. DFO-loaded nanoparticles prepared as described, and $1 \mathrm{ml}$ of the nanosuspension were diluted to $10 \mathrm{ml}$ using PBS. For particle destruction, $0.5 \mathrm{ml}$ of acetonitrile used, and the drug concentration in these samples was determined at $485 \mathrm{~nm} \mathrm{UV}$ spectrophotometric. Based on the result of this assay, an equal concentration drug solution prepared in PBS. Both samples, i.e., loaded- and the free drug mixed by the solution of $\mathrm{FeCl} 3$ $(3.53 \% \mathrm{w} / \mathrm{v}$ in dilute hydrochloric acid (1 in 100), and the resulting mixtures divided into fifteen 1 -ml portions in $1.5 \mathrm{ml}$ polypropylene microtubes. The samples shake vertically $(15 \mathrm{rpm})$ while incubated in $37 \mathrm{oC}$ using a vertically shaking incubator designed and assembled in-house. At the start time of the test and 1, 2, 4, 8, 12, 24, 48, $72 \mathrm{hrs,} 1$ and 2 weeks' intervals, one of the 
aliquots harvested, and $4 \mathrm{ml}$ of double-distilled water added to samples and the absorbance were determined at $485 \mathrm{~nm}$ as a measure of iron-binding. These experiments carried out in triplicate.

\section{Results and Discussion}

\subsection{Preparation of nanoparticles.}

To prepare the chitosan-based nanoparticle, which supported the ability of chitosan in gel formation by making inter and intramolecular linkages, we use the ionotropic gelation method. In this technique, which based on ionic interaction, we attempt to use chitosan as a polycationic polymer and tripolyphosphate as a polyanionic substance. For determining the optimum condition of independent variables and their comparable estimates between real and orthogonal values, the Taguchi experimental design used [39]. In this design, the optimum condition was calculated based on the dominancy of the signal/noise ratio of each factor level. After the initial estimation, the final optimum conditions validated by the preparation of nanoparticle using the particle size analysis in each case. As a result, the optimum setting, indicated earlier in the nanoparticle preparation method, was reliable and highly robust, with the outputs being highly consistent with the theoretical data.

\subsection{In vitro characterization of DFO-loaded nanoparticles.}

The particles obtained in the present study were unidisperse (unimodal curves) with very suitable sizes (below $100 \mathrm{~nm}$ ), in terms of both central tendency indices and dispersity indices for the intended intravenous drug delivery. The final particle size after optimizing the setting was $62 \pm 2.01 \mathrm{~nm}$ (number-based diameter) and 78 $\pm 2.09 \mathrm{~nm}$ (volume-based diameter) for unloaded, and $63 \pm 1 \mathrm{~nm}$ (number-based diameter) and $79 \pm 3.21 \mathrm{~nm}$ (volume-based diameter) for DFO-loaded nanoparticles. Most importantly, all the mean, median, and modal diameters remain without any significant changes after the loading procedure $(\mathrm{P}>0.05)$.

Furthermore, the size dispersity of the nanoparticles population was about the same in DFO-loaded and unloaded nanoparticles. This means that in optimum condition (CS $0.3 \%$ (w/v), TPP $5 \%(w / v)$, TPP/CS $1 / 8$, Temperature $25^{\circ} \mathrm{C}$, Addition time of TPP solution to CS solution 2 minutes, and DFO $6.25 \mathrm{mg} / \mathrm{ml}$ ), the drug loading procedure has no significant effect on particle size and size distribution of the nanoparticles. The particle diameters of about 60 $\mathrm{nm}$ obtained in the current study for DFO-loaded hydrogel nanoparticles remarkably lower compared to 300-400 nm for insulin-loaded hydrogel nanoparticles [10] and >120 nm for ammonium glycyrrhizinate-loaded hydrogel nanoparticles [41], both prepared by ionotropic gelation process. In all these studies, the size of nanoparticles increased when the agent was loaded, while the size of our nanoparticles not significantly changed after DFO loading. Interestingly, the small size of the obtained DFO-loaded nanoparticles in this study is a favorable prerequisite for a long-circulating drug delivery system.

While zeta potential $(\xi)$ measurements provide a critical criterion for the stability of a colloid system, this may be an important parameter for cellular interaction [42]. Although zeta potential measurements have utilized in probing the interaction between cells and biomolecules, thus, it is used to study the interaction between nanoparticles and biological cells $[43,44]$. Therefore, for determining the possible influence of drug loading on surface charge and ionic environmental conditions, we tried to evaluate the zeta potential of nanoparticles before and after loading the drug. In our study, the nanoparticles are undispersed in terms of 
zeta potential, have a wide distribution, and mean zeta potential of close to neutral, but slightly positive. Furthermore, the DFO loading, while shifting the overall mean slightly to positive (from $-1.70 \mathrm{mV}$ for unloaded nanoparticle and $+4.14 \mathrm{mV}$ for DFO loaded nanoparticles), has no observable effect on zeta potential distribution data. Therefore, there seems to be no need for additional surface charge treatment of these particles in order to attain an ideal surface charge in terms of evading the RES in circulation $[5,10,41,45]$.

The shape analysis of a spread of both DFO-loaded and unloaded nanoparticles by transmission electron microscopy (TEM), while highly confirming the laser-based size analysis data, indicated that the prepared nanogels are smoothly spherical with some satellite smaller particles attached to the periphery of each particle. The size and shape uniformity between the particles are evident. Also, again, this test shows that DFO loading has no observable effect of particle size and shape (Fig. 1).

In characterizing the FTIR spectra, originally, there are three determinant peaks of chitosan at around $3447 \mathrm{~cm}^{-1}$ of $\mathrm{OH}, 1066 \mathrm{~cm}^{-1}$ of C-O-C, $1649 \mathrm{~cm}^{-1}$ of $\mathrm{NH}_{2}$. As expected, the peak at 3447 becomes wider as TPP becomes conjugated to chitosan. This event is evident in our spectra and attributed to the resulting enhanced hydrogen bindings, also, in chitosan-TPP nanoparticles peak of $1649 \mathrm{~cm}^{-1}$ of $\mathrm{NH}_{2}$ bending vibrations shifts to around $1570 \mathrm{~cm}^{-1}$. The resulting FTIR spectrum is consistent with the results obtained from the phosphate-modified chitosan film, and this termination can be attributed to the bond between the phosphorus ion and the ammonium. So we suppose that the tri polyphosphoric groups of TPP linked with ammonium groups of chitosan in nanoparticles. Compared with the spectrum of DFO in the spectrum of DFO-loaded nanoparticles, the absorption peak of about $1622 \mathrm{~cm}^{-1}$ (carboxyl group absorption peak) shifts to peak around $1570 \mathrm{~cm}^{-1}$ (salt of carboxyl) appears. The results indicate the presence of the electrostatic interactions between carboxyl groups of DFO and amino groups of chitosan (Fig. 2). Our FTIR results are in agreement with studies published by Yan Wu et al. [41, 46-49].

\subsection{DFO loading parameter \& in vitro drug release.}

The loading parameters of DFO in prepared nanogels at four different drug concentrations displayed in Fig. 3. The loading parameter including loading efficiency, loading amount and loading ratio of $26.1 \% \pm 0.63,8.48 \% \pm 0.021$ and $2.66 \% \pm 1.88$ respectively achieved in optimum conditions which are practically acceptable for the optimum concentration of 6.25 $\mathrm{mg} / \mathrm{ml}$ and notable compared to the other reports in the literature [41, 50, 51] and ensures the feasibility of the method for DFO therapy. Also, our experiments showed no significant adsorption of DFO by the membrane filters used in the study in the experimental conditions, which ensures the data validity obtained upon filtration of the samples throughout the experiments.

The release profiles of DFO from hydrogel nanoparticles while being shaken at $37^{\circ} \mathrm{C}$ are shown in Fig. 4. Drug release from nanoparticles and subsequent biodegradation is essential for developing successful formulations [52]. The amount of drug release from nanoparticles depends on the following: i) Desorption of the surface adsorbed drug; ii) Diffusion through the nanoparticles matrix; iii) Diffusion through the polymer wall (in case of nanocapsules); iv) Nanoparticle matrix erosion, and v) A combined erosion/diffusion process [53].

In the case of matrix devices such as the one, we prepared in this study, because of the uniform dispersion of drug in the matrix, the release occurs by matrix diffusion or matrix erosion [52]. If the drug diffusion is faster than the matrix degradation, then the mechanism of 
drug release occurs mainly by Fickian diffusion. Otherwise, it depends upon the degradation rate $[3,54]$. Considering that we have performed the drug release experiments directly on the nanosuspension, as prepared, without the replacement of the medium, in order to avoid any shock because of the external conditions on the nanoparticles, and also considering the drugloaded ratio in nanodispersion, the following facts can found that the initial release is explosive in the first 24 hours of sampling, including about $60 \%$ of the loaded drug. This release rate becomes gradually lower, followed by a linear phase between the second and seventh days, including about $20 \%$ of the drug. The burst release hypothetically caused by a portion of the drug-associated weakly to nanoparticles, e.g., adsorbed onto the surface of the microsphere. The terminal zero-order release, however, seems to be related to a portion of the drug either bound to nanoparticles more strongly, e.g., via electrostatic associations or entrapped deeply inside the nanogel structures.

\subsection{Stability of DFO loaded nanoparticles.}

According to the results obtained from the particle stability test (data were not shown), nanoparticle sizes remained relatively unchanged at least for three days both in refrigerator and room condition, with no significant difference between these two temperatures. A slight, but significant increase in sizes was evident in both temperatures from one week onward to reach precipitation in one month. This article enlargement behavior could be a result of the hydration and swelling of the nanogels, which ultimately leads to the particle disintegration. It seems that the particles are stable enough in a suspended state for practically critical times before their application time. For further stabilization, as we will see in this study, freeze-drying is a proper solution. Besides, the temperature was shown not to be an essential factor in particle disintegration kinetic. The minor difference in volume-based sizes compared to the numberbased data reflects the appearance of some out of range particles, presumably because of the limited aggregation between nanoparticles.

\subsection{Preparation of nanopowder.}

From a series of tests using simple sugars or non-ionic surfactants as cryoprotectants, the best result, i.e., re-gaining of the particle sizes of the nanoparticles after reconstitution, was obtained with $5 \%(\mathrm{w} / \mathrm{v})$ concentration of glucose. The average particle size after reconstitution was $90 \mathrm{~nm}$. Using 5\% (w/v) of glucose, the appearance of the cake (the dried material) does not become as a regular white powder, i.e., hardened nor fluffy, which is the best for a freezedried formulation. When other cryo-protectants used, the appearance of the dried material was translucent and sticky. Dispersion of the nanoparticles was possible with other agents but as a form of visible aggregates. The good cryoprotective results with glucose probably arise from its ability to bind with water molecules in the amorphous phase during the freezing step. A part of the water in the frozen glucose remained non-frozen (even $32 \% \mathrm{w} / \mathrm{w}$ ), which, in turn, acts as a spacing matrix and plasticizer, thus reducing the pressure of ice crystals against the nanoparticles and preventing harmful aggregation caused by frozen materials.

\subsection{Yield of nanoparticles.}

The data shows that about 25 percent of the solid materials entering the loading procedure other than the cryo-protectant, glucose, remain within the nanosuspension. In other 
words, about $75 \%$ of the dry materials leave the process as the out-of-range solids after the centrifuge, and the overall recovery (accuracy of the processed) was $66.48 \% \pm 4.7$.

\subsection{Iron Binding Capacity.}

The IBCs of DFO-loaded nanoparticles and the equal concentration free DFO solution in PBS shown in Fig. 5. As a direct measure of the functional efficiency of the drug-loaded in nanoparticles in comparison to the free DFO, we designed and carried out a simple test as the exposure of the equal concentration DFO in two states, i.e., DFO-loaded nanoparticles vs. free $\mathrm{DFO}$, to $\mathrm{Fe} 3+$. The results showed that while the free drug maintains its activity (IBC) constant at least for three weeks, which is consistent to the drug stability (previous section), the DFOloaded nanoparticles start from slightly lower IBC, but show a slowly progressing IBC to reach an IBC value of about $50 \%$ higher than the simultaneous free drug samples in two days (Pvalue $<0.05$ ), with a slowly decreasing trend to overlap to the free drug curve. This observation shows the higher efficiency of DFO in iron-binding in Nanoparticles loaded state, which stays for a longer time. The fact that the IBC curves overlap after 72 hours (Pvalue>0.05) maybe because of either the free iron penetration the Nanoparticles after some this time or the migration of the drug to accessible particle surface at this time, an issue deserving more detailed studies beyond the scope of this study.

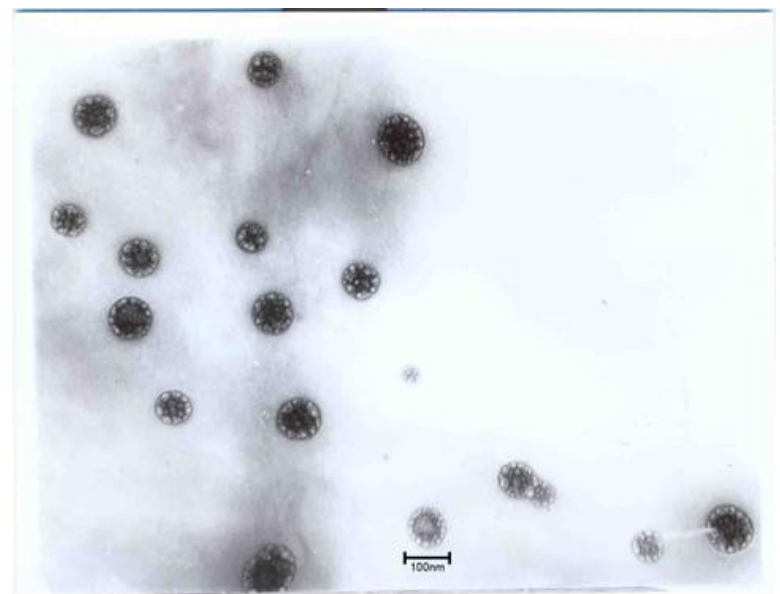

Figure 1. TEM images of optimized DFO loaded nanogels $(6.25 \mathrm{mg} / \mathrm{ml})$. (copyright $@ 2020$ John Wiley \& Sons Inc, License Number:4834270628218, all right reserved).

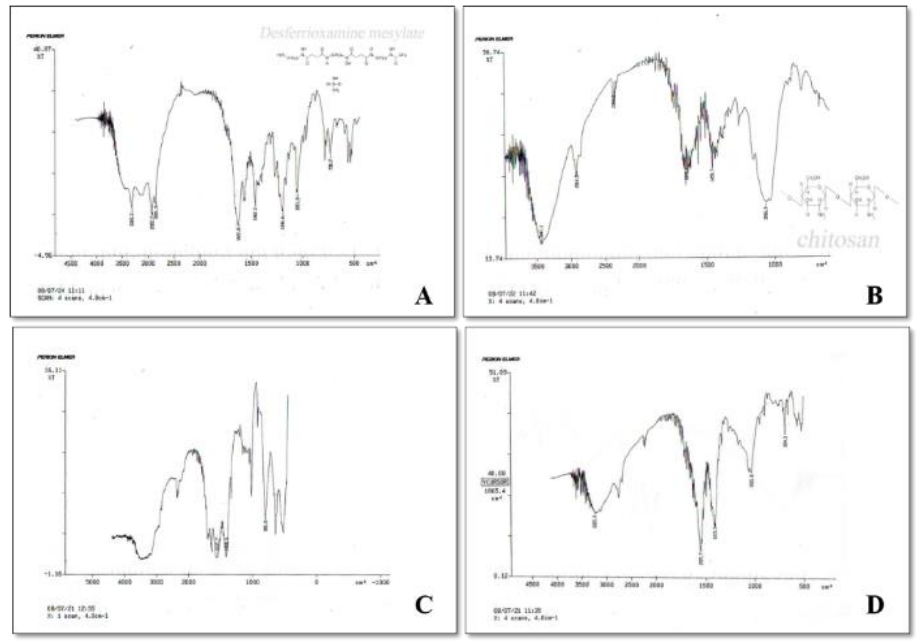

Figure 2. FTIR spectra of DFO (A), Chitosan (B), unloaded nanoparticles (C) and DFO-loaded nanoparticles (D). 

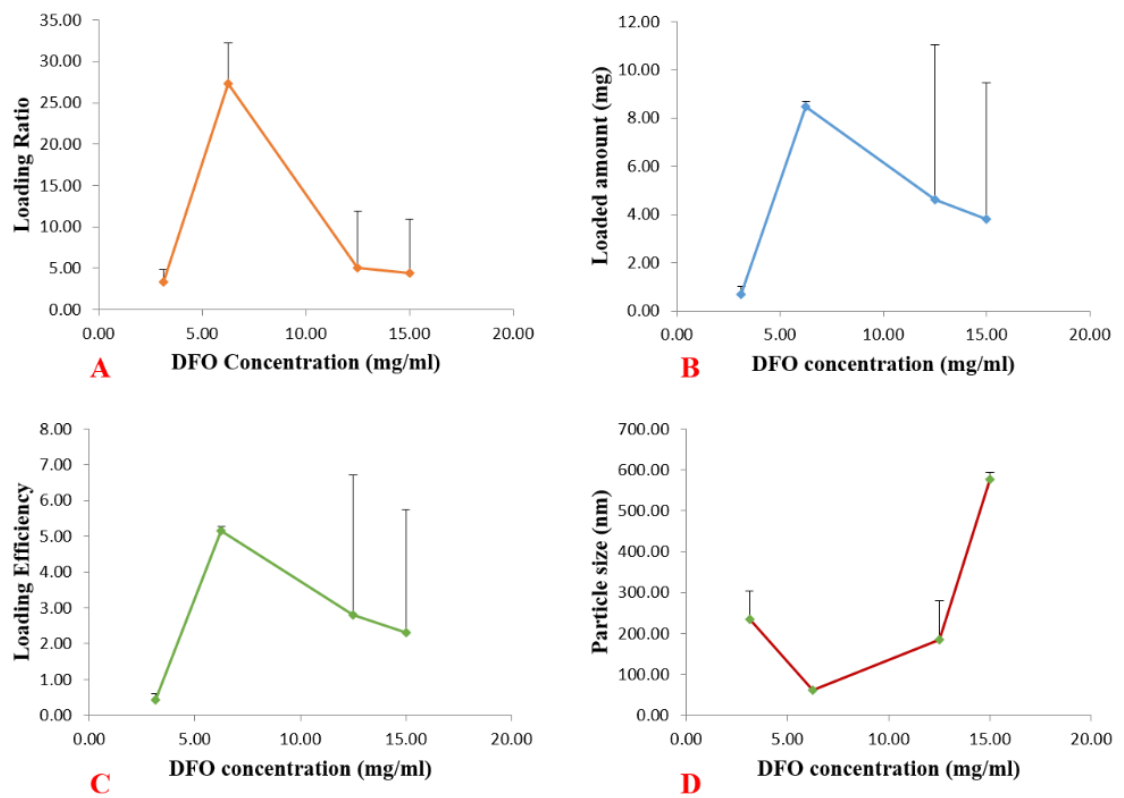

Figure 3. The effects of DFO concentration on Loading ratio (A), Loading efficiency (B), Particle size diameter (C), and Loading amounts (D) of optimized DFO loaded nanogels.

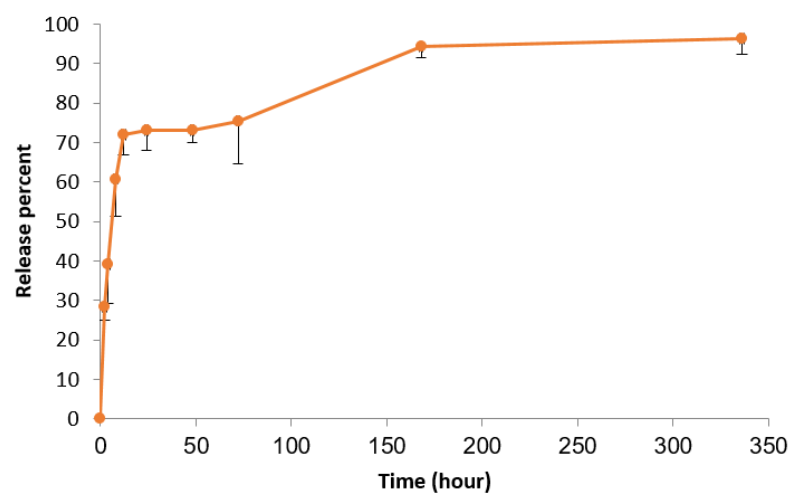

Figure 4. The release profile of optimized DFO loaded nanogels.

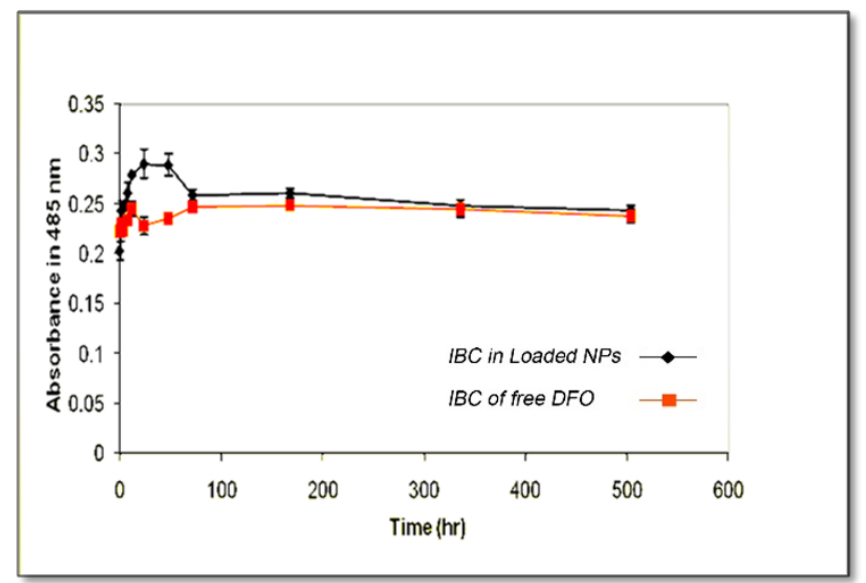

Figure 5. The IBC of optimized DFO loaded nanogels in comparison with unloaded nanoparticles.

\section{Conclusions}

Hydrogel nanoparticles (nanogels) loaded by DFO was prepared, optimized, and characterized in vitro after an extensive fractional factorial design test on the DFO-loaded nanoparticles, based on an ionotropic gelation method using chitosan as the polymer and tripolyphosphate (TPP) as the counter-ion. The determinant parameters optimized in terms of 
the particle size as the central index. Then the drug concentration used during the process was optimized concerning drug loading parameters. Finally, the optimal DFO-loaded nanoparticles characterized in vitro to drug release, particle size, zeta potential, iron-binding capacity, particle stability, and FTIR spectrometry of DFO-loaded nanoparticles, TEM analysis, and freeze-drying of the nanoparticles. Overall the results were indicative of nanocarriers with promising loading capacity, release characteristics, particle yield, and other in vitro tests. Our particles have followed characteristics: very high hydrophilicity, above 90 percent is water; Ideal particle size of about $60 \mathrm{~nm}$ with suitable dispersion and no rare significant particles/associations; Reasonable zeta potential of about $+4.14 \mathrm{mv}$, Spherical uniform shapes, which are desired as a neutral condition to escape from the RES.; FTIR spectrum evidencing the formation of weak chemical associations resulting in nanoparticles formation; according to the results, maybe this interaction was at the surface of the nanocarriers. The capability of being reversibly freeze-dried with minimum deviations from initial particle sizes upon re-dispersion. Therefore, the resulting DFO-loaded nanogels are proper candidates to enter the in vivo efficiency tests. This designed nanoparticle system could be a good candidate for AD by the nose to the brain drug delivery approaches.

\section{Funding}

This work is supported by the School of Pharmacy and Pharmaceutical Sciences Research Center, Shiraz University of Medical Sciences (Grant No. 94-01-05-9115Vice-Chancellery of Research and Technology).

\section{Acknowledgments}

This research has no acknowledgment.

\section{Conflicts of Interest}

The authors declare no conflict of interest.

\section{References}

1. Hamidi, M.; Ashrafi, H.; Azadi, A. Surface functionalized hydrogel nanoparticles. Biomedical materials and diagnostic devices 2012, 191-213, https://doi.org/10.1002/9781118523025.ch6.

2. Narayanaswamy, R.; Torchilin, V.P. Hydrogels and their applications in targeted drug delivery. Molecules 2019, 24, https://doi.org/10.3390/molecules24030603.

3. Lin, C.C.; Metters, A.T. Hydrogels in controlled release formulations: network design and mathematical modeling. Advanced drug delivery reviews 2006, 58, 1379-1408, https://doi.org/10.1016/j.addr.2006.09.004.

4. Jahromi, L.P.; Panah, F.M.; Azadi, A.; Ashrafi, H. A mechanistic investigation on methotrexate-loaded chitosan-based hydrogel nanoparticles intended for CNS drug delivery: Trojan horse effect or not? International journal of biological macromolecules 2019, 125, 785-790, https://doi.org/10.1016/j.ijbiomac.2018.12.093.

5. Gaur, U.; Sahoo, S.K.; De, T.K.; Ghosh, P.C.; Maitra, A.; Ghosh, P.K. Biodistribution of fluoresceinated dextran using novel nanoparticles evading reticuloendothelial system. International journal of pharmaceutics 2000, 202, 1-10, https://doi.org/10.1016/S0378-5173(99)00447-0

6. Soppimath, K.S.; Aminabhavi, T.M.; Kulkarni, A.R.; Rudzinski, W.E. Biodegradable polymeric nanoparticles as drug delivery devices. Journal of controlled release 2001, 70, 1-20, https://doi.org/10.1016/S0168-3659(00)00339-4

7. Moghimi, S.M.; Hunter, A.C.; Murray, J.C. Long-circulating and target-specific nanoparticles: theory to practice. Pharmacological reviews 2001, 53, 283-318.

8. Agnihotri, S.A.; Mallikarjuna, N.N.; Aminabhavi, T.M. Recent advances on chitosan-based micro- and nanoparticles in drug delivery. Journal of Controlled Release 2004, 100, 5-28, https://doi.org/10.1016/j.jconrel.2004.08.010. 
9. Wang, J.; Cheng, X.; Zhang, X.; Liu, G.; Wang, Y.; Zhou, W.; Zhang, Y. A combination of indomethacin and atorvastatin ameliorates cognitive and pathological deterioration in PrP-hA $\beta$ PPswe/PS1 $\Delta \mathrm{E} 9$ transgenic mice. Journal of neuroimmunology 2019, 330, 108-115, https://doi.org/10.1016/j.jneuroim.2019.03.003

10. Gan, Q.; Wang, T. Chitosan nanoparticle as protein delivery carrier-systematic examination of fabrication conditions for efficient loading and release. Colloids and Surfaces B: Biointerfaces 2007, 59, 24-34, https://doi.org/10.1016/j.colsurfb.2007.04.009.

11. Cardia, M.C.; Carta, A.R.; Caboni, P.; Maccioni, A.M.; Erbì, S.; Boi, L.; Meloni, M.C.; Lai, F.; Sinico, C. Trimethyl Chitosan Hydrogel Nanoparticles for Progesterone Delivery in Neurodegenerative Disorders. Pharmaceutics 2019, 11, https://doi.org/10.3390/pharmaceutics11120657.

12. Kisby, B.; Jarrell, J.T.; Agar, M.E.; Cohen, D.S.; Rosin, E.R.; Cahill, C.M.; Rogers, J. T.; Huang, X. Alzheimer's Disease and Its Potential Alternative Therapeutics. Journal of Alzheimer's disease \& Parkinsonism 2019, 9.

13. Wang, X.; Guan, F. Therapeutic Intranasal Delivery for Alzheimer's Disease. In: Therapeutic Intranasal Delivery for Stroke and Neurological Disorders. Springer: 2019; pp 117-133, https://doi.org/10.1007/9783-030-16715-8_10.

14. Fine, J.M.; Kosyakovsky, J.; Baillargeon, A.M.; Tokarev, J.V.; Cooner, J.M.; Svitak, A.L.; Faltesek, K.A.; Frey, W.H.; Hanson, L.R. Intranasal deferoxamine can improve memory in healthy C57 mice, suggesting a partially non-disease-specific pathway of functional neurologic improvement. Brain and Behavior 2020, https://doi.org/10.1002/brb3.1536.

15. Porter, J.B. Practical management of iron overload. British journal of haematology 2001, 115, 239-252, https://doi.org/10.1046/j.1365-2141.2001.03195.x.

16. Cecil, R.L.; Goldman, L.; Bennett, J.C. Pocket companion to cecil textbook of medicine. Saunders: 2001.

17. Duscher, D.; Trotsyuk, A.A.; Maan, Z.N.; Kwon, S.H.; Rodrigues, M.; Engel, K.; Stern-Buchbinder, Z.A.; Bonham, C.A.; Barrera, J.; Whittam, A.J. Optimization of transdermal deferoxamine leads to enhanced efficacy in healing skin wounds. Journal of Controlled Release 2019, 308, 232-239, https://doi.org/10.1016/j.jconrel.2019.07.009.

18. Holden, P.; Nair, L.S. Deferoxamine: an angiogenic and antioxidant molecule for tissue regeneration. Tissue Engineering Part B: Reviews 2019, 25, 461-470, https://doi.org/10.1089/ten.teb.2019.0111.

19. Akıncıoğlu, H.; Gülçin, İ. Potent Acetylcholinesterase Inhibitors: Potential Drugs for Alzheimer's Disease. Mini reviews in medicinal chemistry 2020, 20, https://doi.org/10.2174/1389557520666200103100521.

20. Tan, E. C. K.; Johnell, K.; Bell, J. S.; Garcia-Ptacek, S.; Fastbom, J.; Nordström, P.; Eriksdotter, M. Do Acetylcholinesterase Inhibitors Prevent or Delay Psychotropic Prescribing in People with Dementia? Analyses of the Swedish Dementia Registry. The American Journal of Geriatric Psychiatry 2020, 28, 108117, https://doi.org/10.1016/j.jagp.2019.06.008.

21. Yang, Y.H.; Liscic, R.; Dominguez, J. Framework of treating Alzheimer's dementia. Brain Science Advances 2019, 5, 82-93, https://doi.org/10.1177/2096595820902580.

22. Then, C.K.; Liu, K.H.; Liao, M.H.; Chung, K.H.; Wang, J.Y.; Shen, S.C. Antidepressants, sertraline and paroxetine, increase calcium influx and induce mitochondrial damage-mediated apoptosis of astrocytes. Oncotarget 2017, 8, https://doi.org/10.18632/oncotarget.23302.

23. Ali, M.M.; Ghouri, R.G.; Ans, A.H.; Akbar, A.; Toheed, A. Recommendations for Anti-inflammatory Treatments in Alzheimer's Disease: A Comprehensive Review of the Literature. Cureus 2019, 11, https://doi.org/10.7759/cureus.4620.

24. Agam, G.; Levine, J. Neuroprotective effects of lithium in neuropsychiatric disorders. In: Neuroprotection in Autism, Schizophrenia and Alzheimer's Disease. Elsevier: 2020; pp 209-241, https://doi.org/10.1016/B978-0-12-814037-6.00006-9.

25. Hara, Y.; McKeehan, N.; Dacks, P.; Fillit, H.M. Evaluation of the neuroprotective potential of Nacetylcysteine for prevention and treatment of cognitive aging and dementia. Evaluation 2017, 4, 201-206, http://dx.doi.org/10.14283/jpad.2017.22.

26. Kile, S.; Au, W.; Parise, C.; Sohi, J.; Yarbrough, T.; Czeszynski, A.; Johnson, K.; Redline, D.; Donnel, T.; Hankins, A. Reduction of Amyloid in the Brain and Retina After Treatment With IVIG for Mild Cognitive Impairment. American Journal of Alzheimer's Disease \& Other Dementias ${ }^{\circledR}$ 2020, 35, https://doi.org/10.1177/1533317519899800.

27. Relkin, N.R.; Thomas, R.G.; Rissman, R.A.; Brewer, J.B.; Rafii, M.S.; Van Dyck, C.H.; Jack, C.R.; Sano, M.; Knopman, D.S.; Raman, R. A phase 3 trial of IV immunoglobulin for Alzheimer disease. Neurology 2017, 88, 1768-1775, https://doi.org/10.1212/WNL.0000000000003904.

28. Liu, J.; Wang, M. Carvedilol protection against endogenous A $\beta$-induced neurotoxicity in N2a cells. Cell Stress and Chaperones 2018, 23, 695-702, https://doi.org/10.1007/s12192-018-0881-6.

29. Nikseresht, S.; Bush, A.I.; Ayton, S. Treating Alzheimer's disease by targeting iron. British journal of pharmacology 2019, 176, 3622-3635, https://doi.org/10.1111/bph.14567.

30. Dekens, D.W.; De Deyn, P.P.; Sap, F.; Eisel, U.L.M.; Naudé, P.J.W. Iron chelators inhibit amyloid- $\beta$ induced production of lipocalin 2 in cultured astrocytes. Neurochemistry international 2020, 132, https://doi.org/10.1016/j.neuint.2019.104607. 
31. Khoshnoud, M.J.; Siavashpour, A.; Bakhshizadeh, M.; Rashedinia, M. Effects of sodium benzoate, a commonly used food preservative, on learning, memory, and oxidative stress in brain of mice. Journal of biochemical and molecular toxicology 2018, 32, https://doi.org/10.1002/jbt.22022.

32. Borah P, Kuma MVS, Computational investigation on the role of C-Terminal of human albumin on the dimerization of A $\beta 1-42$ peptide, Biointerface Research in Applied Chemistry, 2020, 10, 1, $4944-4955$, https://doi.org/10.33263/BRIAC101.944955

33. Rashedinia, M.; Lari, P.; Abnous, K.; Hosseinzadeh, H. Protective effect of crocin on acrolein-induced tau phosphorylation in the rat brain. Acta Neurobiol Exp 2015, 75, 208-219.

34. Fine, J.M.; Forsberg, A.C.; Stroebel, B.M.; Faltesek, K.A.; Verden, D.R.; Hamel, K.A.; Raney, E.B.; Crow, J.M.; Haase, L.R.; Knutzen, K.E. Intranasal deferoxamine affects memory loss, oxidation, and the insulin pathway in the streptozotocin rat model of Alzheimer's disease. Journal of the neurological sciences 2017, 380, 164-171, https://doi.org/10.1016/j.jns.2017.07.028

35. Zhang, Y.; He, M.L. Deferoxamine enhances alternative activation of microglia and inhibits amyloid beta deposits in APP/PS1 mice. Brain research 2017, 1677, 86-92, https://doi.org/10.1016/j.brainres.2017.09.019

36. Zhang, Y.; Fan, B.Y.; Pang, Y.L.; Shen, W.Y.; Wang, X.; Zhao, C.X.; Li, W.X.; Liu, C.; Kong, X.H.; Ning, G.Z. Neuroprotective effect of deferoxamine on erastin-induced ferroptosis in primary cortical neurons. Neural Regeneration Research 2020, 15, https://doi.org/10.4103/1673-5374.274344.

37. Farthing, K.; Ferill, M.J.; Generally, J.A.; Jones, B.; Sweet, B.V.; Mazur, J.N. Drug Facts \& Comparisons. Pocket Version 2006, 9.

38. Hardman, J.; Limbird, L.E. Goodman and Gilman's the pharmacological basis of therapeutics. Mc GrawHill, United States of America: 2001.

39. Hamidi, M.; Azadi, A.; Ashrafi, H.; Rafiei, P.; Mohamadi-Samani, S. Taguchi Orthogonal Array Design for the Optimization of Hydrogel Nanoparticles for the Intravenous Delivery of Small-Molecule Drugs. Journal of Applied Polymer Science 2012, 126, 1714-1724, https://doi.org/10.1002/app.36778

40. Hamidi, M.; Azadi, A.; Mohamadi-Samani, S.; Rafiei, P.; Ashrafi, H. Valproate-Loaded Hydrogel Nanoparticles: Preparation and Characterization. Journal of Applied Polymer Science 2012, 124, 4686-4693, https://doi.org/10.1002/app.35527.

41. Taguchi, G.; Chowdhury, S.; Wu, Y. Taguchi's quality engineering handbook. John Wiley \& Sons Hoboken: 2005.

42. Zhang, Y.; Yang, M.; Park, J.H.; Singelyn, J.; Ma, H.; Sailor, M.J.; Ruoslahti, E.; Ozkan, M.; Ozkan, C. A Surface Charge Study on Cellular Uptake Behavior of $\mathrm{F}_{3}$-Peptide-Conjugated Iron Oxide Nanoparticles. small 2009, 5, 1990-1996, https://doi.org/10.1002/smll.200900520.

43. Al-Kassas, R.; Wen, J.; Cheng, A.E.M.; Kim, A.M.J.; Liu, S.S.M.; Yu, J. Transdermal delivery of propranolol hydrochloride through chitosan nanoparticles dispersed in mucoadhesive gel. Carbohydrate polymers 2016, 153, 176-186, https://doi.org/10.1016/j.carbpol.2016.06.096.

44. Park, S.; Hamad-Schifferli, K. Evaluation of hydrodynamic size and zeta-potential of surface-modified Au nanoparticle-DNA conjugates via Ferguson analysis. The Journal of Physical Chemistry C 2008, 112, 76117616, https://doi.org/10.1021/jp8004426.

45. R Nayak, B Nanda, A review on formulation, design of nanostructured material through oil-in-water microemulsion, Letters in Applied NanoBioScience, 2020, 9, 2, $945 \quad-\quad 951$, https://doi.org/10.33263/LIANBS92.945951

46. Moataza, M.S. Chelating Ability of the Chitosan-glucan Complex from Aspergillus niger NRRL595 Biomass Recycling in Citric Acid Production. Research Journal of Agriculture and Biological Sciences 2006, 2, 132-136.

47. Singh, A.; Narvi, S.S.; Dutta, P.K.; Pandey, N.D. External stimuli response on a novel chitosan hydrogel crosslinked with formaldehyde. Bulletin of Materials Science 2006, 29, 233-238, https://doi.org/10.1007/BF02706490.

48. Nada, A.M.A.; El-Sakhawy, M.; Kamel, S.; Eid, M.A.M.; Adel, A.M. Effect of Chitosan and its Derivatives on the Mechanical and Electrical Properties of Paper Sheets. Egyptian Journal of Solids 2005, 28.

49. Palpandi, C.; Shanmugam, V.; Shanmugam, A. Extraction of chitin and chitosan from shell and operculum of mangrove gastropod Nerita (Dostia) crepidularia Lamarck. International Journal of Medicine and Medical Sciences 2009, 1, 198-205.

50. Pan, Y.; Li, Y.; Zhao, H.; Zheng, J.; Xu, H.; Wei, G.; Hao, J.; Cui, F. Bioadhesive polysaccharide in protein delivery system: chitosan nanoparticles improve the intestinal absorption of insulin in vivo. International journal of pharmaceutics 2002, 249, 139-147, https://doi.org/10.1016/S0378-5173(02)00486-6.

51. Ko, J.A.; Park, H.J.; Hwang, S.J.; Park, J.B.; Lee, J.S. Preparation and characterization of chitosan microparticles intended for controlled drug delivery. International journal of pharmaceutics 2002, 249, 165174, https://doi.org/10.1016/S0378-5173(02)00487-8.

52. Freitas Mariano, K.C.; Monteiro do Nascimento, M.H.; Querobino, S.M.; Ramos Campos, E.V.; de Oliveira, J.L.; Yokaichiya, F.; Franco, M.K.K.D.; Alberto-Silva, C.; de Paula, E.; Lombello, C.B. Influence of chitosan-tripolyphosphate nanoparticles on thermosensitive polymeric hydrogels: structural organization, drug release mechanisms and cytotoxicity. International Journal of Polymeric Materials and Polymeric Biomaterials 2020, 69, 592-603, https://doi.org/10.1080/00914037.2019.1596909. 
53. Lee, J.H.; Yeo, Y. Controlled drug release from pharmaceutical nanocarriers. Chemical engineering science 2015, 125, 75-84, https://doi.org/10.1016/j.ces.2014.08.046.

54. Peppas, N.A.; Bures, P.; Leobandung, W.; Ichikawa, H. Hydrogels in pharmaceutical formulations. European journal of pharmaceutics and biopharmaceutics 2000, 50, 27-46, https://doi.org/10.1016/S09396411(00)00090-4. 